\title{
Interactive Error Repair for an Online Handwriting Interface
}

\author{
Wolfgang Huerst, Jie Yang, Alex Waibel \\ Interactive Systems Laboratories \\ Carnegie Mellon University \\ School of Computer Science \\ Pittsburgh, PA 15213-3890, USA
}

\{wolfgang+, yang+, waibel $\} @$ cs.cmu.edu

\begin{abstract}
Current online handwriting recognition systems have very limited error recovery mechanisms. In this paper, we discuss the problem of error repair in an online handwriting interface. Based on user study of common repair patterns found in human handwriting, we propose an approach that allows users to recover from recognition errors. The basic idea is to handle the error repair at the interface level by interacting with users. The method requires few modifications on original recognition engine and imposes few restrictions on users. We have developed a prototype system to demonstrate the proposed concept and perform user study when the system provides error recovery mechanisms.
\end{abstract}

\section{Keywords}

Online handwriting recognition, error repair in human handwriting, error recovery, interactive user interface

\section{INTRODUCTION}

Pen-based handwriting input provides an alternative way for human-computer interaction. However, it is impossible to develop a perfect handwriting recognition system. Schomaker reported that even humans were unable to achieve a recognition accuracy of $100 \%$ (Schomaker, 1994). Our studies also discovered that recognition errors result from not only the system but also human mistakes such as misspelled words (Hurst, 1997). Therefore, even with a "perfect" recognition engine we would still face the problem of misinterpretation. On the other hand, instead of pursuing a perfect recognition system, it is possible to develop a system with reasonable user's satisfaction by providing error recovery mechanisms. Research in speech recognition (Suhm, et al. 1996) has demonstrated that even with unreliable baseline spoken language interpretation technology it is possible to significantly reduce the time to interact with a system via spoken language by various error repair strategies. In this paper, we investigate the problem of error repair in an on-line handwriting recognition system. The objective is to develop concepts that could minimize the user's efforts to recover from errors in an on-line handwriting interface. While most current pen-based systems only offer repair possibilities by providing additional buttons (e.g., a "clear"-button) or by recognizing a fixed set of gestures, we aim at providing users with a handwriting interface that can recover recognition errors in natural and flexible ways.

\section{ERROR AND ERROR REPAIR}

Based on user studies performed by Schomaker (Schomaker, 1994), human handwritten materials contain different types of errors. These errors include: discrete noises, badly formed shapes, input that is legible by humans but not by the algorithm; badly spelled words; words that are unsolicited in the data collection process, canceled material, and device generated errors. A handwritten input legible to humans is not necessarily legible to a recognition engine and vise versa. In order to learn more about error repair patterns in an online handwriting recognition system, we studied a database of 3466 single words and 3410 text sequences each containing about eight words. We found that about $13 \%$ of the words and $23 \%$ of the word sequences contained errors. We further discovered that users tried to do corrections sometimes even though they were not asked to do any correction. Based on the user study, we can define three types of common repair patterns in an online handwriting system:

- Deletions, e.g., scratched out letters

- Completions and Insertions, e.g., adding some strokes to a previously written letter

- Overwriting, e.g., overwriting a wrong letter

These three types of error repair cover most of error repairs in an online handwriting recognition system. However, it is a challenging problem to handle these repair patterns in a handwriting recognition system. In the next section, we propose an interactive approach to minimizing such an effort on system modification. 


\section{INTERACTIVE ERROR REPAIR}

In an online handwriting recognition system, the input contains not only spatial information but also time information. Time information helps to increase recognition rate. But sometimes it also causes problems, such as delayed tstrokes and i- or j-dots. In fact, delayed t-strokes and i- or j-dots can be considered as repairs. In this way we can use a unified framework to handle repair and delayed strokes. However, identifying the repair mode of performing the required repair is much more difficult than that of handling the delayed-strokes. We cannot use simple heuristics like those used for the delayed strokes. For example, it is impossible to determine if a delayed stroke is a t-stroke or a repair gesture that crosses out the letter "l" without the use of context information and feedback from the recognition engine. Since there is a great variation in repair patterns, it is very difficult for a recognition engine to handle all the repair patterns. In other words, a general recognition engine cannot take care of all these variations without reorganizing the complete structure of the system and recognition algorithms. In order to solve this problem, we propose to handle error repair at the preprocessing level. In fact, by interacting with the user, the system can handle repair at the preprocessing level without any feedback from the recognizer. The goal of the repair handling algorithms is to transform an input trajectory $S$, which may contain error repair actions, into a clean trajectory $S^{*}$. This process is done at the interface level by interacting with the user. The trajectory $S^{*}$ is input to the recognition engine.

A major problem in handling error repair is to detect repair actions, i.e., detect which part of input trajectory contains repair. In order to detect repair actions, we introduce some specific parameters in the current handwriting recognition system (Hurst, 1997). If these parameters change, it is assumed that a repair occurs. For example, a repair is assumed, if the leftmost $x$-coordinates value between two locals extreme is much smaller than the ones written before. After a repair is detected some simple heuristics are used to classify the repair type, i.e., to determine if it is a deletion, a completion/insertion, or an overwriting. By directly indicating the result of the repair handling we try to influence users repair behavior in a way that his/her actions can be easier detected and classified. For example, if a user deletes some parts of a word and the system misinterpret this repair action, the user will repeat his/her repair actions because no repair has been indicated. We have designed some heuristics in the way that is able to detect such "repeated" repair patterns (Hurst, 1997). Figure 1 shows the repair handling scheme.

\section{EVALUATION}

In order to evaluate the proposed approach, we have developed a prototype system using the NPen++, an online handwriting recognition system developed in our lab (Manke et al. 1995). We collected some repair data with four users. We randomly chose a set of 200 word pairs of equal looking words (e.g., "chair" and "hair") and asked the users to write the first one and to transform/correct the written word into the second one. The data were collected

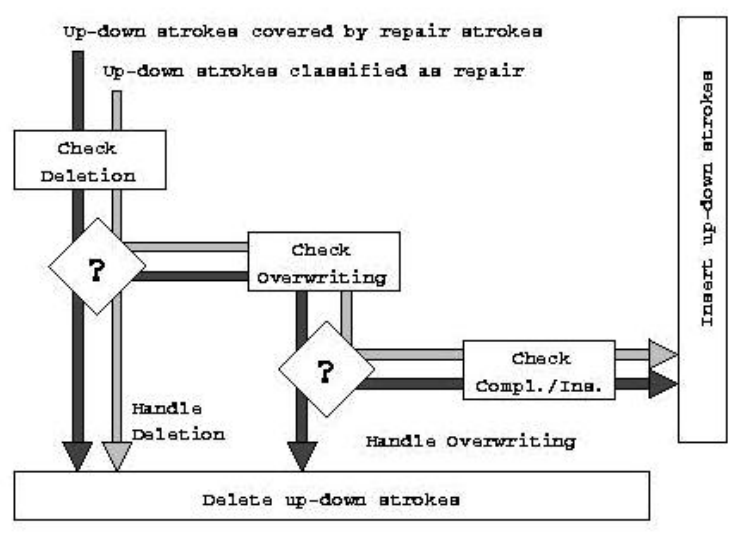

Figure 1. Repair handling scheme

in two times. The system gave no feedback to users in the first collection but indicated the repair results in the second time. The recognition accuracy without the use of any repair handling heuristics was only $8 \%$. With the use of our repair handling heuristics recognition performance increased to $37 \%$. With interactive repair indication the performance was $65 \%$. The recognition accuracy of the baseline system on clean data was $88 \%$.

\section{CONCLUSION}

We have presented an interactive approach to error repair for an online handwriting recognition system. We have demonstrated that, in an online recognition system, the error repair can be effectively handled at the preprocessing level without significantly modifying the recognition engine. We are current working on improving the repair handling schemes. We have also implemented some additional features that allow repair not only in the input signal but in the recognition result. We will perform user studies to evaluate these methods.

\section{REFERENCES}

1. Hurst, W. (1997), "Repair in online handwriting recognition," Master thesis, University of Karlsruhe.

2. Manke, S., Finke, M., and Waibel, A. (1995), "Npen++: A Writer Independent, Large Vocabulary On-Line Cursive Handwriting Recognition System," Proc. of the International Conference on Document Analysis and Recognition.

3. Schomaker, L. R. B. (1994), "User-Interface Aspects in Recognizing Connected-Cursive Handwriting," Proceedings of the IEE Colloquium on Handwriting and Pen-based input. 